\title{
AGILE METHODOLOGY AND APPLICATION OF THE VROOM-YETTON-JAGO MODEL IN METALLURGY
}

\author{
${ }^{1}$ Michal KAVAN, ${ }^{2}$ Lenka ŠVECOVÁ, ${ }^{2}$ Kryštof ŠULC \\ ${ }^{1}$ Czech Technical University in Prague, Faculty of Mechanical Engineering, Prague, Czech Republic, EU, \\ michal.kavan@fs.cvut.cz \\ ${ }^{2}$ Czech Technical University in Prague, Masaryk Institute of Advanced Studies, Prague, Czech Republic, EU, \\ lenka.svecova@cvut.cz, krystof.sulc@cvut.cz
}

https://doi.org/10.37904/metal.2019.996

\begin{abstract}
A brief overview of the status of European steel mills is the basis for the possibility of incorporating the VroomYetton-Jago model in metallurgy. Not only in the Czech Republic, the steel industry is heavily influenced by the emerging applications of Industry 4.0, which will lead to new production standards and, at the same time, the pressure of global competition will increase. The advent of Industry 4.0 applications is the improvement of project management techniques, specifically the requirements for custom software to be compatible with enterprise systems while simplifying work. The use of Agile Approach is suitable for compatible SW development, and there are several decision styles for successful implementation. The Vroom-Yetton-Jago model is appropriate for the business manager to know which decision-making style to follow in a given task. The model, through defined decision-making situations, determines the decision-making style and supports the successful completion of the project and its implementation.
\end{abstract}

Keywords: Vroom-Yetton-Jago Model, Metallurgy, SRUM, Project management, Industry 4.0

\section{INTRODUCTION}

The Czech Republic is not among the European steel powers in terms of its production. But not with meaning. The Czech Republic is also not one of the richest EU countries and thus has limited opportunities to subsidize steel mills in economic difficulties. If the European Parliament is indeed aiming to break the ban on public support in the steel industry, it is expected that the competitive position of Czech steelmakers will deteriorate. In any case, the only way out of an unfavorable situation is to achieve a sustainable top position in technical development in metallurgy. That is, the way of intensively increasing the added value added. In practical terms, this means the ongoing willingness to abolish the highest quality products on the market and to replace them with more refined ones. Radical shortening of innovation cycles, application of previously unprecedented levels of creativity, in meeting rapidly growing environmental protection requirements. For example, in recycling metals, batteries, fluorescent lamps and the like. Czech metallurgists cannot compete with the developing world (or rich EU countries) by producing cheap steel. It must go the way of expanding the production of hightech products. In other words, point to the highest added value [1,2].

The aim of the article is to show how to use the general Vroom-Yetton-Jago model in the metallurgical industry with respect to the current impacts of Industry 4.0. Comprehensive integration of still non-communicating (Vroom-Yetton-Jago model) control systems can help promote real process control. From research, through the stages of technical preparation of production, organizational preparation of production, implementation and, finally, feedback.

\section{IMPROVING PROJECT MANAGEMENT TECHNIQUES IN METALLURGY}

This article focuses on promoting project practices that can assist in the application of the Industrial Revolution 4.0 to metallurgy. This can be done without the use of new devices, hardware, but even faster developing software, giving users real value [3]. 
We have information systems around us everywhere. But in many places too often they work counterproductively. Instead of saving time for users and simplifying their work, they take it from them. They do not add value to customers as expected. Head-to-foot positioning of such control systems is also the task of Industry 4.0. Applying traditional project management has changed radically in the software development world. The traditional approach has replaced the progressive agile methodology of software development project management. Unlike traditional waterfall approaches, agile methodologies (such as SCRUM) require software companies and their development teams to step by step (Sprint to Sprint) to meet user needs (4.0 requirements). The Scrum technique is undoubtedly the best known and most widespread technology in agile software development in the world. Above all, this is our contribution [4].

In the areas of Czech metallurgy, there are high-quality software professionals and companies who are sincerely trying to come up with innovative ideas and the highest quality IT products. Unfortunately, this is not enough for Industry 4.0. Agile methodologies need to be applied to software development and adapted to the specifics of metallurgy management. In Scrum, as an agile software development project management methodology to meet the Industry 4.0 strategy, advanced user requirements need to be regularly refined in each Sprint's Backlog. Today's competitive environment is so rapid that changes in projects and ways of managing them are increasingly necessary and frequent. As a response to this state of frequent implementation of frequent changes in software development, agile methodologies have been developed [5].

The application of agile approaches is suitable for cases where it is not possible to know (and prepare for) at the beginning of a project what the project requires to be reliably incorporated into the new software. With the correct application of the methodology, a "black hole" for money cannot be created, with low productivity, because the implementation phase is divided into many time slots (Sprints) that are passed on to the customer in parts. This creates a continuous series of challenging controls, a series of feasibility studies. There are many different agile planning methodologies. Including their combinations. For example, "Extreme Programming" with its "Planning Game", "Planning Poker", or "Team Velocity" [4,5].

In fact, today's agile approach can be compared to a traditional approach, with a suppressed pre-investment phase. On the other hand, the agile approach greatly enhances monitoring. This is a cyclically and informally feasible administrative feasibility study. In practical life and especially in publicly funded construction, the agile approach is very risky for the investor. It easily leads to poorly solvable and protracted lawsuits. Everything works perfectly when developing software. Each programmer finds his place and the team gets the necessary speed. Properly guided decision support promotes rapid achievement of the desired result. The rule of the narrow spot applies here. The product manager and scrum master must be able to work together and make decisions in the project manager's intentions [4].

The main advantage of agile planning in metallurgy is the ability of the development team to uncover major issues at an early stage of design. The entire implementation team should ideally be involved in the planning process, as each of the highly qualified professionals can bring their unique idea. However, to manage intellectuals is the most challenging issue that has its limits in working discipline. It is best to apply autocratic decision-making primarily in a crisis, when decisions need to be taken quickly, based on experience.

\subsection{About Backlog grooming}

At least once a week, everyone will meet and prepare Backlog together. That means a list of tasks in metallurgy for next week. Deleting unnecessary tasks from the list and compiling a list of new backlog tasks according to customer requirements. It adjusts priorities, estimates workloads, or splits large jobs into smaller, processable iterations. It is based on the release plan and its milestones. Sense of urgency is maintained to keep the team under moderate but constant pressure [3]. 


\subsection{Daily briefing (Stand-up)}

It is dedicated to synchronizing and familiarizing each development team member with the current work status. The implementation team in metallurgy will discuss the planned tasks very briefly. Implementers will provide information: what was done yesterday; what is planned to do today; and the issues that need to be solved $[3,4]$. The meeting is short (maximum 15 minutes). Technical details are not discussed. Possible subsequent professional discussions may occur if individual team members require it.

\subsection{Retrospective}

Held at the end of an iteration. It applies only to members of the implementation team, including Scrum Master, who oversees facilitating the consultation. Facilitation aims to lead the team to the goal, despite obstacles to ineffective communication, misunderstanding and ambiguity. It serves to streamline processes, projects, products and customer relationships. They are focused on generalization of experience and skills of realization team members. The purpose is to uncover the weaknesses of team productivity and eliminate them permanently [3].

\subsection{Scrum Master position requirements in metallurgy}

First and foremost is enthusiasm and interest in the thing. It should be certified at CSM (Certified Scrum Master) or CSPO (Certified Scrum Product Owner) or CSP (Certified Scrum Professional). Furthermore, he / she should have experience with project management in Scrum environment. This knowledge includes qualifications in the areas of coaching, facilitation, change management, leadership, team building, teaching, communication skills, root cause analysis, etc. It should also have an overview of methodologies and philosophies such as: Agile Organization and Culture, Agile Product Ownership, Scrum, Kanban, Lean, Extreme Programming, System Thinking, Scaling and more [4]. In terms of personality traits, he should have the ability to empathy, listen, help others, find new ones, and want to rationalize things.

Each team will focus on its area of complex problem. The resulting running cycle time will be four weeks (see Figure 1) (weekly Sprints, times number of teams). The metallurgy is specific, compared to IT, where project durations are often longer, and especially four-week sprints are inadequate. The output of one team is the entry of the Sprint of the other team, and in this way the set amount is dispatched when it passes through all Scrum teams. Each team defines a list of tasks that they must complete before their output serves the Sprint next team.

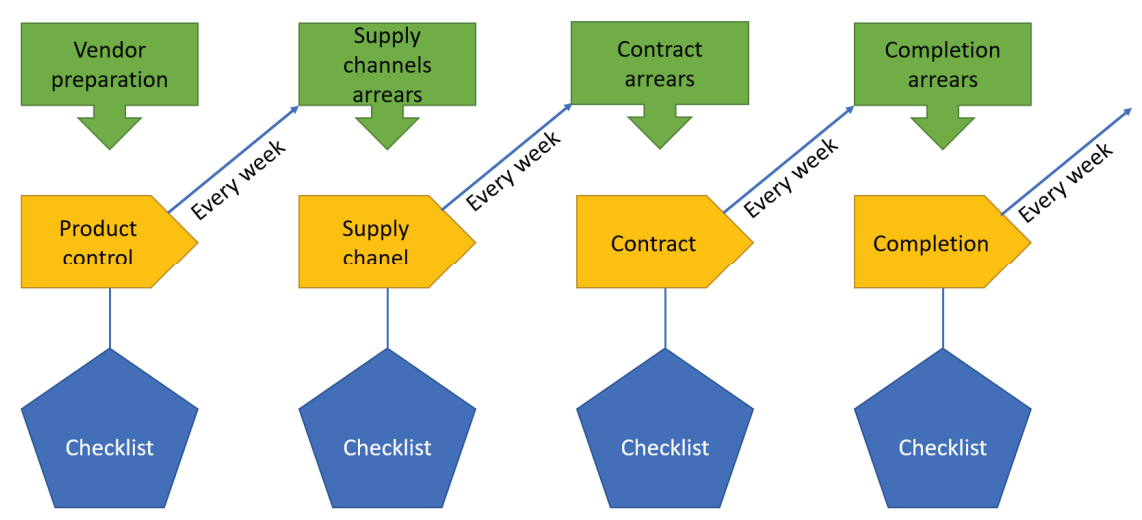

Figure 1 Scheme of the proposed solution [own study]

\section{APPLICATION OF VROOM-YETTON-JAGO DECISION MODEL IN METALLURGY}

Rationality of decision-making is a central part of process management. The position of a corporate manager must be applied in increasingly complex organizational structures. In our case of agile project management for 
the development of new software to implement the requirements of the Industrial Revolution 4.0, it is primarily the decision of the three main characters: Product Manager, Product owner and Scrum Master [6].

They must consciously or subconsciously make many decisions in a variety of situations and conditions. Above all, the decision of the product manager, the product owner and the Scrum Master must lead to business success. It is well known that the decision maker must solve his business problems [6]:

1) Define

2) Collect information and data

3) Consider options

4) Choose the best options

5) Schedule execution

6) Evaluate the result

7) Move further decision-making to a higher level

However, the product manager, product owner, or Scrum master in metallurgy must be able to decide whether he decides autocratically alone or will be able to consult the problem or even let the implementation team decide for himself. Of course, when developing software, we come across many decision-making situations and several decision criteria. There may also be time for a decision. And the severity requirements of decisions will generally also vary, depending on the conditions. So how to make the right decision? Autocratic or democratic [7].

To find out, it is extremely appropriate to use the Vroom-Yetton-Jago model in metallurgy. It is based on the claim that the choice is conditioned by a specific situation and has simplified and mapped many of these situations. The model therefore leads the decision maker to choose the right decision-making style for his team leadership. It is a normative model that works with five different decision-making styles. There are two autocratic styles: (1) Autocratic Al - The leader will make information easily available; (2) Autocratic All - the leader decides based on further information from followers in the group, then decides himself. The model allocates two autoreactive-consultative styles: (3) Consultative $\mathrm{Cl}$ - A leader shares a problem with followers, seeks ideas and information before deciding; and (4) Consultative KII - the leader shares the problem with the group members together but decides himself. Finally, the model specifies one cooperative style: (5) Team GII teamwork - team decision. The leader (can continue to act as a coach) will meet the group to discuss the situation. This supports joint efforts. It's more time consuming. The leader focuses and directs the discussion but does not decide for himself. The final decision is taken by the whole group [6-8].

The Vroom-Yetton-Jago model serves as a map through which I translate my path that leads to a decisionmaking style based on the experience of Vroom-Yetton-Jago (autocratic - democratic group). For a model to work, the product manager, product owner, or Scrum master must answer the following seven questions. They relate to the quality of decisions, commitment, information about the problem, and information related to decision-making. Finally, using the Yes / No answers, a solution can be found on the right side of the model. So, to what extent to involve the followers in the decision [8].

For a left-to-right model path, we are looking for an $\mathrm{A} / \mathrm{N}$ answer to the following questions [6]:

- Is there a quality requirement? Is the nature of the solution crucial? Are there technical or rational reasons to choose between possible solutions?

- Do I have enough information to decide on quality?

- Is the problem structured? Are Alternative Procedures and Methods for Their Evaluation known?

- Is the adoption of decisions subordinate to its implementation?

- If I made this decision myself, is it reasonably certain that my subordinates would accept it?

- Subordinates share the organizational objectives to be achieved in solving this problem?

- Is there a likelihood of conflict between subordinates in obtaining a preferred solution? 
Based on the answers, the individual styles can be found in the graph (see Figure 2).

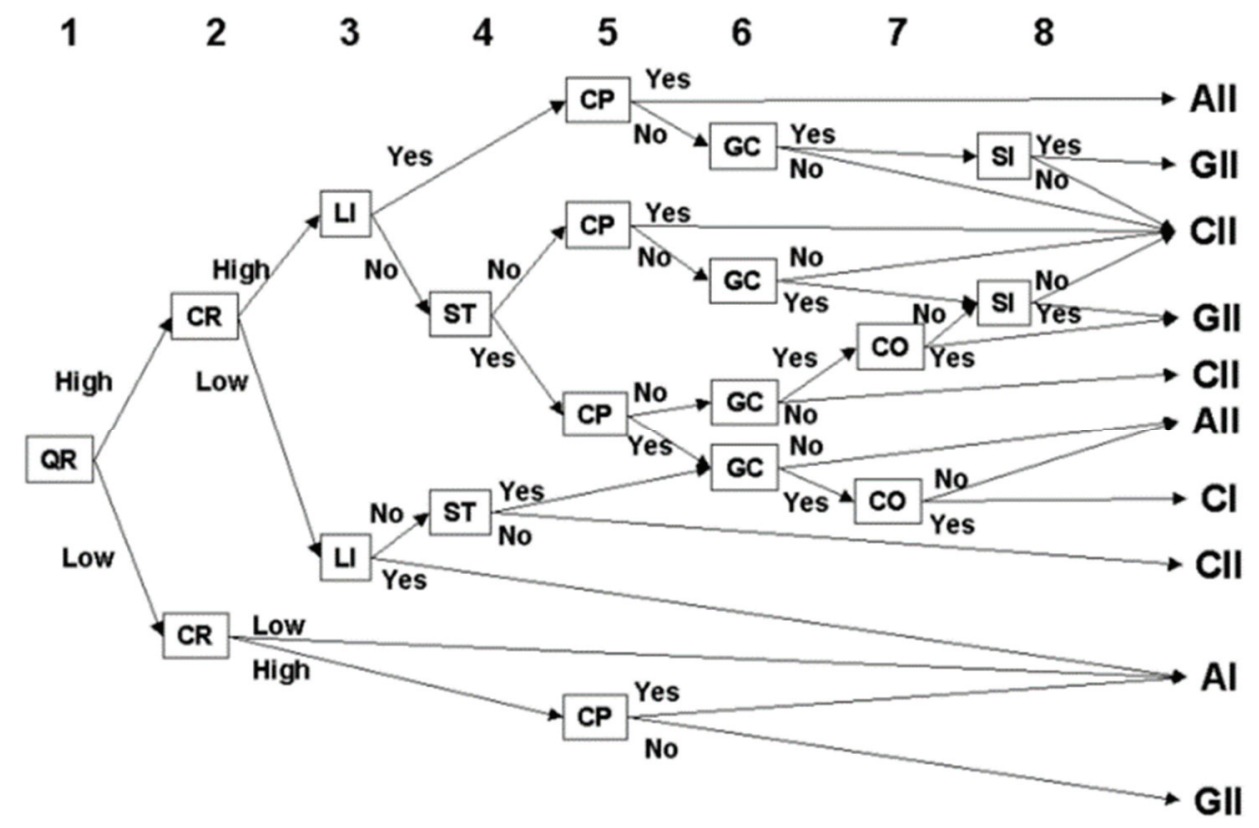

Figure 2 Individuals styles [9]

Individual questions 1-7 have a diagnostic character and are shown as decision tree nodes. Although some questions cannot be answered with absolute certainty, the solver must choose one of the options.

This theory gives the manager guidance on how to successfully and quickly analyses the problem and choose the right decision style. The model assumes that a decision style that has been successful in a situation may not be successful for others. Therefore, the manager's decision-making style should not be monotonous, but should be flexible in responding to the situation.

Different versions have appeared in the world since the Vroom-Yetton-Jago model. However, they retain the original decision and management styles ( $\mathrm{Al}, \mathrm{All}, \mathrm{KI}, \mathrm{KII}, \mathrm{SII}$ ) and evaluation criteria.

It is therefore possible to customize the model, customize it. For example, in the direction of relative decisionmaking, starting with some time-consuming penalties.

\section{CONCLUSION}

Nowadays, when the impact of Industry 4.0 is to digitize many processes in companies, including production lines, it is necessary to consider implementing new approaches in the context of organizational culture and appropriate methods. Metallurgy is generally more specific to traditional cultures, but the digitization of both production and metallurgy will inevitably lead to cooperation with high-tech companies and the interconnection of machine implementations with the move to virtualization and digitization. Putting such solutions into operation is necessary to perform rather agile methodology (such as Scrum).

The Vroom-Yetton-Jago model offers five decision-making styles that are all well applicable in the metallurgical industry, but communication with high-tech vendors will generally abstain from first-level decision-making styles (autocratic or consultative). Scrum methodology, which is suitable for the introduction of digitization of production, basically does not count on autocratic styles or autocratic elements in consultative styles. It is based on teamwork. This fact, however, will face the culture of several traditional engineering and metallurgical companies. It is therefore essential that management in these sectors develop the competence of workers in this direction. 
If the decision maker has little time, he will choose rather authoritative style. Conversely, if it is more concerned with the motivation of subordinate workers than with time savings, it chooses a more participative style in metallurgy.

To put it simply, it is clear from the model that a common decision-making style is preferable if:

- You need a lot of information from others.

- $\quad$ The problem cannot be easily defined.

- This is a challenging goal.

- You have enough time to manage the group's decisions.

To put it simply, it can also be seen from the model that an autocratic style is appropriate if:

- The decision maker has a better overview of the situation than others.

- The decision maker is sure to decide for himself.

- The team will take decisions without dissatisfaction.

- There is a crisis and there is little time.

But with this model, the decision problem can be properly grasped. Because the model is very illustrative, quickly applicable and delivers effective results. It gives clear recommendations in metallurgy.

The main drawbacks are that the model does not distinguish between peace, i.e. it requires only $\mathrm{A} / \mathrm{N}$ responses.

\section{REFERENCES}

[1] EUROFER (Employment data); Oxford Economics estimates based mainly on Eurostat's 'Annual detailed enterprise statistics for industry'. [viewed 2019-05-21]. Available from: https://bami.bg/media/docs/eurofer.pdf

[2] COCCIA, Mario. Steel market and global trends of leading geo-economic players. Int. J. Trade and Global Markets. 2014. vol. 7, no. 1, pp. 36-52.

[3] RANDRUP, Nils, DRUCKENMILLER Douglas and BRIGGS, Robert O. Philosophy of Collaboration. 49th Hawaii International Conference on System Sciences (HICSS) [online]. IEEE, 2016. 2016. p. 898-907 [viewed 2019-0521]. DOI: $10.1109 / \mathrm{HICSS} .2016 .115$. ISBN 978-0-7695-5670-3. Available from: http://ieeexplore.ieee.org/document/7427292/

[4] Cocco, Luisanna, et al. Simulating kanban and scrum vs. waterfall with system dynamics. In: International conference on agile software development. Springer, Berlin, Heidelberg, 2011. pp. 117-131.

[5] AUGUSTINE, Sanjiv, et al. Agile project management: steering from the edges. Communications of the ACM. 2005. vol. 48 , no. 12 , pp. $85-89$.

[6] LEICHEROVA, Veronika, and Martin JANUSKA. Recommendations for the Selection of the Appropriate DecisionMaking Style for the Selected Problem Situations Using the Vroom-Yetton-Jago Model. In: VISION 2020: Innovation, Development, Sustainability and Economic Growth, vol. 1-3. Vienna, 2013. pp. 908-920.

[7] BROWN, F. William; FINSTUEN, Kenn. The use of participation in decision making: A consideration of the vroomyetton and vroom-jago normative models. Journal of Behavioral Decision Making. 1993. vol. 6, no. 3, pp. 207-219.

[8] FIELD, Richard HG; ANDREWS, J. P. Testing the incremental validity of the Vroom-Jago versus Vroom-Yetton models of participation in decision making. Journal of Behavioral Decision Making, 1998, vol. 11, no. 4, pp. 251 261.

[9] VROOM, V.H., JAGO, A.G. The new leadership: Managing participation in organizations. Englewood Cliffs, NJ: Prentice Hall, 1988. 